\title{
Efficient sorting of quantum-optical wave packets by temporal-mode interferometry
}

\author{
D. V. Reddy ${ }^{1}$, M. G. Raymer ${ }^{1, *}$, C. J. McKinstrie ${ }^{2}$ \\ ${ }^{1}$ Oregon Center for Optics and Department of Physics, University of Oregon, Eugene, Oregon 97403, USA \\ ${ }^{2}$ Bell Laboratories, Alcatel-Lucent, Holmdel, New Jersey 07r33, USA \\ *Corresponding author: raymer@uoregon.edu
}

Compiled September 19, 2018

\begin{abstract}
Long-distance quantum communication relies on storing and retrieving photonic qubits in orthogonal field modes. The available degrees of freedom for photons are polarization, spatial-mode profile, and temporal/spectral profile. To date, methods exist for decomposing, manipulating, and analyzing photons into orthogonal polarization modes and spatial modes. Here we propose and theoretically verify the first highly efficient method to carry out analogous operations for temporally and spectrally overlapping, but field-orthogonal, temporal modes. The method relies on cascaded nonlinear-optical quantum frequency conversion. (C) 2018 Optical Society of America

OCIS codes: 270.5565, 190.4223, 060.1155, 060.4230
\end{abstract}

Photons - elementary excitations of the electromagnetic field - have four degrees of freedom: helicity and three components of momentum (which determines frequency through a dispersion relation). In a beam-like geometry these may be stated as polarization, spatial-mode profile, and frequency. In order to fully utilize light for transmitting information in an optical network, either classical or quantum, it is necessary to be able to sort a beam of light according to the states of photons associated with each degree of freedom. Sorting states of light by polarization, center frequency (wavelength), or spatial-mode profile (transverse parity $[1,2]$ or orbital angular momentum [3-5]) are now commonplace. In quantum networks, other single-photon encoding implementations include the 'dual-rail qubit' [6] and the 'time-bin qubit' [7].

A longstanding unsolved problem has been the sorting of light according to arbitrary states of frequency. By Fourier transformation, such states can be represented in the time domain, thereby introducing the idea of 'temporal modes' (TMs) - a set of orthogonal functions of time that form a complete basis for representing an arbitrary state in the frequency degree of freedom [8]. TM multiplexing does not offer increased theoretical bounds on channel capacity when compared to WDM. However, it does offer performance increases for quantum networks. Efficient sorting and manipulation of temporal modes would enable concurrent usage of communication pathways by variously encoded photonic qubits in different 'TM-channels', as well as make available a new basis for encoding quantum information in light - i.e. the 'temporal-mode qubit.' Since the dimensionality of the TM-state space is in principle infinite, single photons could encode quantum information as multidimensional quantum bits, or qudits, which would enable increased channel capacity by multimode-entanglement enabled teleportation [9] and device-independent quantum key distribution based on loophole-free Bell inequality viola- tions [10]. In addition, optical TMs at the same central frequency, spatial mode and polarization co-propagate over long distances and remain orthogonal even in the presence of dispersion.

Here we propose and theoretically verify the first scheme that can sort TMs with near-100\% efficiency, making TM sorting a viable tool for optical communication. We use an approach based on the observation that quantum frequency conversion (QFC) is TM-mode selective, as first pointed out by Eckstein et al. [11] and McGuinness et al. [12]. In QFC, the signal field of interest interacts with one (or two) strong laser pulse(s) in a second (or third)-order nonlinear-optical medium, such as a crystalline waveguide or a silica optical fiber [13]. The shapes of the laser pulses determine which TM in the signal will be selected. QFC in materials possessing $\chi^{(2)}$ or $\chi^{(3)}$ nonlinearity is accomplished using three-wave mixing (TWM) [14-17] or four-wave mixing (FWM) Bragg scattering [12,18-20] respectively. Earlier proposed implementations of QFC by TWM [11] used a single stage of QFC, which cannot exceed a natural limit for TM 'selectivity' of about $0.8[11,21,22]$. The selectivity of a third-order nonlinear-optical single-stage version driven by two pump laser pulses is limited by nonlinear phase-modulation to about 0.65 (see Fig. 1). Our proposed scheme, which we call temporal-mode interferometry (TMI), uses cascaded stages of QFC, and can provide close to $100 \%$ TM selectivity, opening the door for manipulation of TM qubits or multi-level qudits. The method may also be used for TM channel multiplexing in classical optical telecom.

To model the QFC process, we designate the participating frequency channels by the letters $s, r$ for the signal photons and $p$ (and $q$ ) for the strong pump field(s). We denote normalized field envelopes in the $j$-channel by $A_{j}(z, t)$ and the group slowness (inverse group velocity) by $\left.\beta_{j}^{\prime} \equiv[d \beta / d \omega]\right|_{\omega=\omega_{j}}$, and we neglect higher-order dispersion, which is valid for narrow-band pulses. Then the 
equations of motion for QFC are $[18,23]$ :

$$
\begin{aligned}
\left(\partial_{z}+\beta_{p}^{\prime} \partial_{t}\right) A_{p} & =i(\gamma / 2) \delta_{F}\left[\left|A_{p}\right|^{2}+2\left|A_{q}\right|^{2}\right] A_{p} \\
\left(\partial_{z}+\beta_{q}^{\prime} \partial_{t}\right) A_{q} & =i(\gamma / 2) \delta_{F}\left[2\left|A_{p}\right|^{2}+\left|A_{q}\right|^{2}\right] A_{q} \\
\left(\partial_{z}+\beta_{r}^{\prime} \partial_{t}\right) A_{r} & =i \gamma A_{p} A_{q}^{*} A_{s} \\
& +i \gamma \delta_{F}\left[\left|A_{p}\right|^{2}+\left|A_{q}\right|^{2}\right] A_{r} \\
\left(\partial_{z}+\beta_{s}^{\prime} \partial_{t}\right) A_{s} & =i \gamma A_{p}^{*} A_{q} A_{r} \\
& +i \gamma \delta_{F}\left[\left|A_{p}\right|^{2}+\left|A_{q}\right|^{2}\right] A_{s},
\end{aligned}
$$

where the self- and cross-phase modulation factor $\delta_{F}$, is 0 for TWM and 1 for FWM. $\gamma$ is the product of the effective $\chi^{(2)}$ or $\chi^{(3)}$ nonlinearity and the square roots of the pump-pulse energies. For TWM, $A_{q}(z, t) \equiv 1$. The solution for Eq. (1) can be written in terms of Green functions $G_{i j}\left(t, t^{\prime}\right)$. For $j \in\{r, s\}$ and medium length $L$ :

$$
A_{j}(L, t)=\int_{-\infty}^{\infty} \mathrm{d} t^{\prime} \sum_{k=r, s} G_{j k}\left(t, t^{\prime}\right) A_{k}\left(0, t^{\prime}\right) .
$$

The four kernels $G_{i j}\left(t, t^{\prime}\right)$ comprise a generalized beam-splitter transformation, representing frequency conversion or non-conversion of each of the two possible input fields of distinct carrier frequencies [24].

For each $G_{i j}\left(t, t^{\prime}\right)$, we can perform numerically a singular-value decomposition (SVD), also called a Schmidt decomposition, into a set of singular-value functions (temporal Schmidt modes) with associated singular values (Schmidt coefficients), which we denote by $\rho_{n}$ and $\tau_{n}$, which satisfy $\left|\rho_{n}\right|^{2}+\left|\tau_{n}\right|^{2}=1$. $\left|\rho_{n}\right|^{2}$ is the frequency conversion efficiency $(\mathrm{CE})$ of the $n^{\text {th }}$ mode, and can be interpreted as the probability of QFC in the case of single-photons. The modes are numbered in decreasing order of the Schmidt coefficients. We denote by $\phi_{n}(t)$ the $s$-input modes, and $\Psi_{n}(t)$ are $r$-output modes. In addition, there are $r$-input modes $\psi_{n}(t)$ and $s$-output modes $\Phi_{n}(t)$. All the Green functions can be expressed in terms of these four mode sets as follows [24]:

$$
\begin{aligned}
& G_{r r}\left(t, t^{\prime}\right)=\sum_{n=1}^{\infty} \tau_{n} \Psi_{n}(t) \psi_{n}^{*}\left(t^{\prime}\right), \\
& G_{r s}\left(t, t^{\prime}\right)=\sum_{n=1}^{\infty} \rho_{n} \Psi_{n}(t) \phi_{n}^{*}\left(t^{\prime}\right), \\
& G_{s r}\left(t, t^{\prime}\right)=-\sum_{n=1}^{\infty} \rho_{n} \Phi_{n}(t) \psi_{n}^{*}\left(t^{\prime}\right), \\
& G_{s s}\left(t, t^{\prime}\right)=\sum_{n=1}^{\infty} \tau_{n} \Phi_{n}(t) \phi_{n}^{*}\left(t^{\prime}\right) .
\end{aligned}
$$

The chief objective is to design a QFC device that can selectively frequency convert or 'drop' the first Schmidt mode with unit efficiency, whilst allowing $100 \%$ unconverted transmission of all orthogonal modes. The principal figure of merit for such a drop device is the 'selectivity,' [22] defined as $S=\left|\rho_{1}\right|^{4} / \sum_{j=1}^{\infty}\left|\rho_{j}\right|^{2}$.
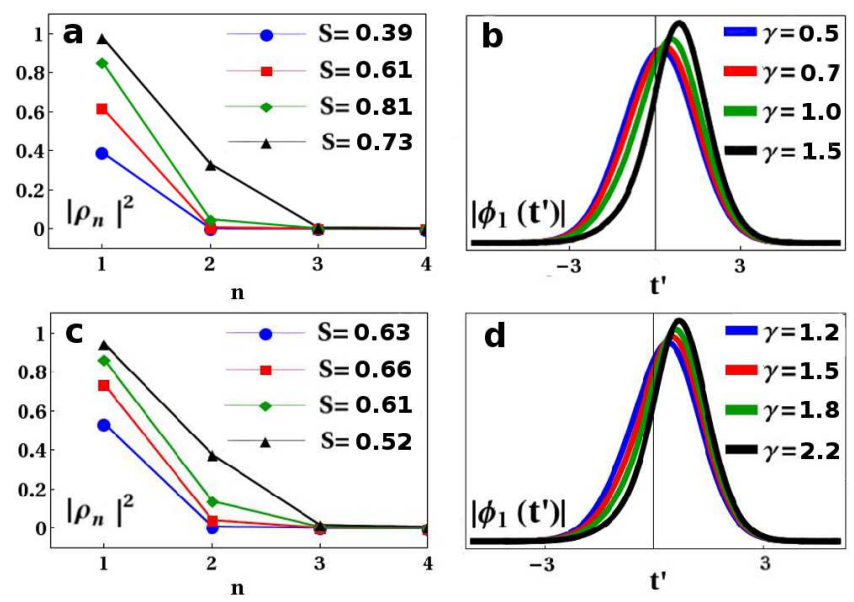

Fig. 1. Single-stage TWM (a,b) and FWM (c,d) dominant Schmidt-mode conversion efficiencies $\left|\rho_{n}\right|^{2}$ (a,c) with selectivities $S$ listed in the legend. (b,d) $s$-channel input Schmidt modes. Pump- $p$ was Gaussian in shape.

The best previous attempts at optimizing selectivity using a single-stage QFC scheme have converged on frequency choices that are group-velocity matched, namely $\beta_{p}^{\prime}=\beta_{s}^{\prime} \neq \beta_{r}^{\prime}\left(=\beta_{q}^{\prime}\right)[18,19,22]$. Eq. (1) been fully solved analytically for this case for TWM [22]. In addition, for optimum results in FWM, the medium is required to be long enough for a complete inter-pump-pulse collision (no-overlap to no-overlap) [18]. However, both TWM and FWM schemes have encountered limits that are universal to generic systems governed by coupled-mode equations such as Eq. (1), and have yielded selectivities limited to around 0.8 and Schmidt-mode profiles that are severely temporally skewed relative to the groupvelocity-matched pump shapes (Fig. 1). The limit to single-stage selectivity and the Schmidt-mode temporalskewness occur due to oscillatory distortion of the Green functions at higher $\gamma$ akin to Burnham-Chiao ringing $[22,25]$. These distortions are minimal at lower CE (Fig. 1).

We have discovered that using a two-stage interferometric scheme overcomes both of these limitations. Each stage is configured for $50 \% \mathrm{CE}$ for the first Schmidt mode (Fig. 2), and functions as a 50/50 beam splitter with the $r$ - and $s$-frequency channels representing its two input and output arms. An $s$-input photon in the first Schmidt mode in the first stage will be partially coherently frequency converted into the $r$-channel with a phase picked up from the pump field(s). If all fields are allowed to participate in QFC in the second stage at the right relative phases, the two effective beam splitters will function as a frequency-shifting Mach-Zehnder interferometer, allowing for complete FC or complete back-coversion of the photon. The effect operates only on the first Schmidt mode, as the higher-order modes have negligible $\mathrm{CE}$ in both stages. We call the scheme 'temporal-mode interferometry.' There are two configurations for which the four pulsed fields interact in both stages: 1) 'reversed 


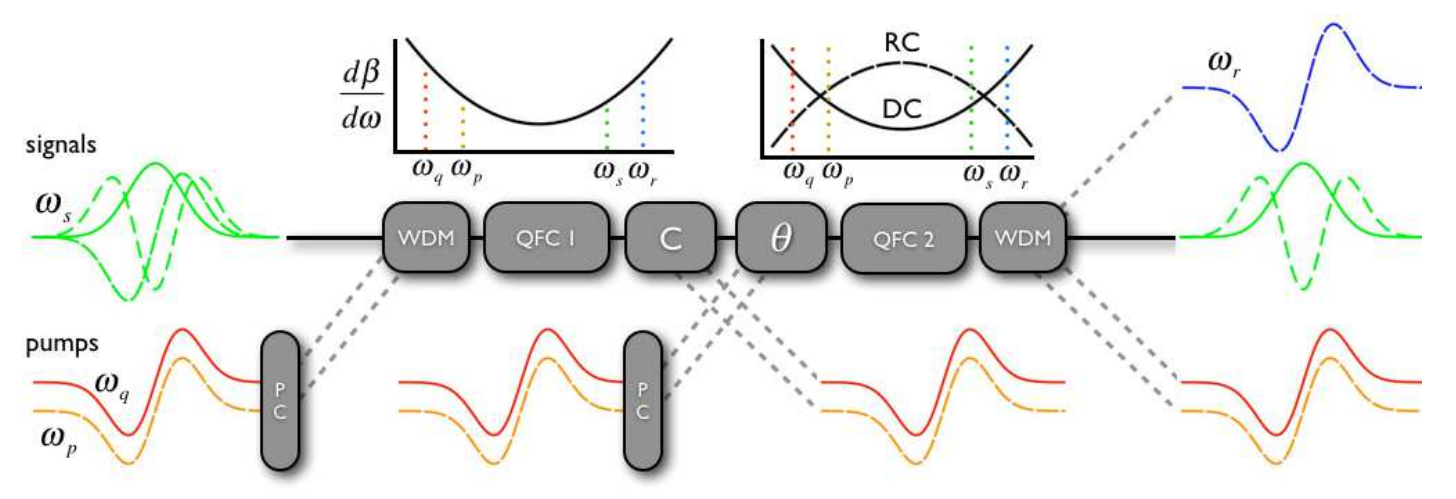

Fig. 2. Temporal-mode interferometer using two nonlinear media (QFC 1 and QFC 2) with identical (DC) or opposite-sign (RC) dispersion. By choosing the pump-pulse shapes, pump powers and phase shift $\theta$, one can selectively frequency convert a specific $s$ (green) TM into an $r$ (blue) TM at a different central frequency, whilst not affecting temporally-orthogonal $s$-input TMs. The $q$-channel is used only for $\chi^{(3)}$ implementations. WDM stands for wavelength-division multiplexer. PC stands for pre-chirp module, which are necessary for $\chi^{(3)}$ implementations. The coupler C contains frequency dependent delays for the DC case.

collision' (RC), in which the second-stage dispersion is inverted relative to the first, i.e. $\left.\beta_{r}^{\prime}\right|^{(1)}=\left.\beta_{s}^{\prime}\right|^{(2)}$ and vice versa, such that the relative speeds of the pulses are reversed, and 2) 'double collision' (DC), in which the second-stage dispersion is identical to the first, but the fast pulses are time delayed relative to the slow pulses in between the stages so that they walk through each other again.

For the two-stage TMI, the combined Green function kernel $G_{r s}\left(t, t^{\prime}\right)$ is given by the interferometric equation:

$$
\begin{array}{r}
G_{r s}\left(t, t^{\prime}\right)=\int_{-\infty}^{\infty} \mathrm{d} t^{\prime \prime}\left[G_{r s}^{(2)}\left(t, t^{\prime \prime}\right) G_{s s}^{(1)}\left(t^{\prime \prime}, t^{\prime}\right)\right. \\
\left.+e^{i \theta} G_{r r}^{(2)}\left(t, t^{\prime \prime}\right) G_{r s}^{(1)}\left(t^{\prime \prime}, t^{\prime}\right)\right],
\end{array}
$$

where the parenthetical superscripts indicate the stage. A control phase $\theta$ is externally applied to any one of the channels in between stages. Equation (4) illustrates the need for inter-stage temporal mode matching between the output Schmidt modes of the first stage and the input Schmidt modes of the second stage for the scheme to work. For TWM-TMI, this is best achieved by the RC configuration. Figure 3(a)-3(e) presents the $\mathrm{CE}$ and Schmidt modes for the RC configuration TWMTMI with $\zeta=\left|\beta_{r}^{\prime}-\beta_{s}^{\prime}\right| L / \tau_{p}=200$, where $L$ is the per stage medium length and $\tau_{p}$ is the pump width. The selectivity was computed to be $0.9846\left(\left|\rho_{1}\right|^{2}=0.9975\right.$, $\left.\left|\rho_{2}\right|^{2}=0.0110\right) . \zeta$ is also the ratio of time-widths of $r$ and $s$-Schmidt modes (Fig. 3(b)-3(e)). The selectivity of the RC-TWM configuration improves asymptotically with $\zeta$. A $\zeta$ of 200 can be realized, for example, in a typical 5-cm long PPLN waveguide with a 70-fs pump-pulse and signal wavelengths at $795 \mathrm{~nm}$ and $1324 \mathrm{~nm}$.

The first-stage output pulses have the correct relative time delays for the second-stage input in the $\mathrm{RC}$ configuration. However, TWM-TMI can also be implemented in the DC configuration, using appropriate frequency dependent delay lines in between stages. For $\zeta=200$, it yielded a slightly lower selectivity of 0.9805 $\left(\left|\rho_{1}\right|^{2}=0.9957,\left|\rho_{2}\right|^{2}=0.0134\right)$ due to relatively inferior inter-stage mode-matching. TWM-TMI can be easily extended to an arbitrary number of stages with arbitrary ordering of RC/DC stage-interfaces and appropriately lower pump powers. Such schemes will be explored in a later publication.

Nonlinear phase-modulation in FWM severely affects the Schmidt-mode phase-profiles, and restricts FWMTMI to the RC configuration. The pumps must be prechirped with specific phase-profiles [18] for each stage to enhance inter-stage mode-matching. Figure 3(f)-3(o) shows the numerically computed CE, Schmidt-mode amplitudes, and phases corresponding to FWM-TMI with selectivity $0.9873\left(\left|\rho_{1}\right|^{2}=0.9973,\left|\rho_{2}\right|^{2}=0.0082\right)$. The pump pre-chirps (Fig. $3(\mathrm{k})$ ) were specifically chosen to yield flat phase profiles for the r-output (Fig. $3(\mathrm{~m})$ ) and $s$-input (Fig. 3(n)) Schmidt modes. The results were computed for medium lengths that were long enough for complete pump-pulse collisions in each stage $\left(\left|\beta_{r}^{\prime}-\beta_{s}^{\prime}\right| L /\left(\tau_{p}+\tau_{q}\right)=5\right)$, a condition easily satisfied by use of highly nonlinear fibers for QFC [12]. FWMTMI, besides yielding good selectivity, gives the ability to choose the $s$ - and $r$-channel Schmidt-mode shapes independently of each other (by reshaping both pumps), allowing for efficient mode-selective single-photon temporal reshaping. FWM-TMI, in contrast to TWM-TMI, does not enforce a high temporal-width ratio between the signal channels. However, nonlinear phase-modulation hinders the extension of FWM-TMI into multi-stage schemes.

In conclusion, TMI can selectively manipulate photonic temporal mode components of any shape accessible by our ability to reshape strong pump pulses. Modular TMI devices also possess a phase control $(\theta)$ that can suppress QFC completely and make the device transparent on demand. The addition of temporal-mode analysis and multiplexing to polarization and OAM analysis and 

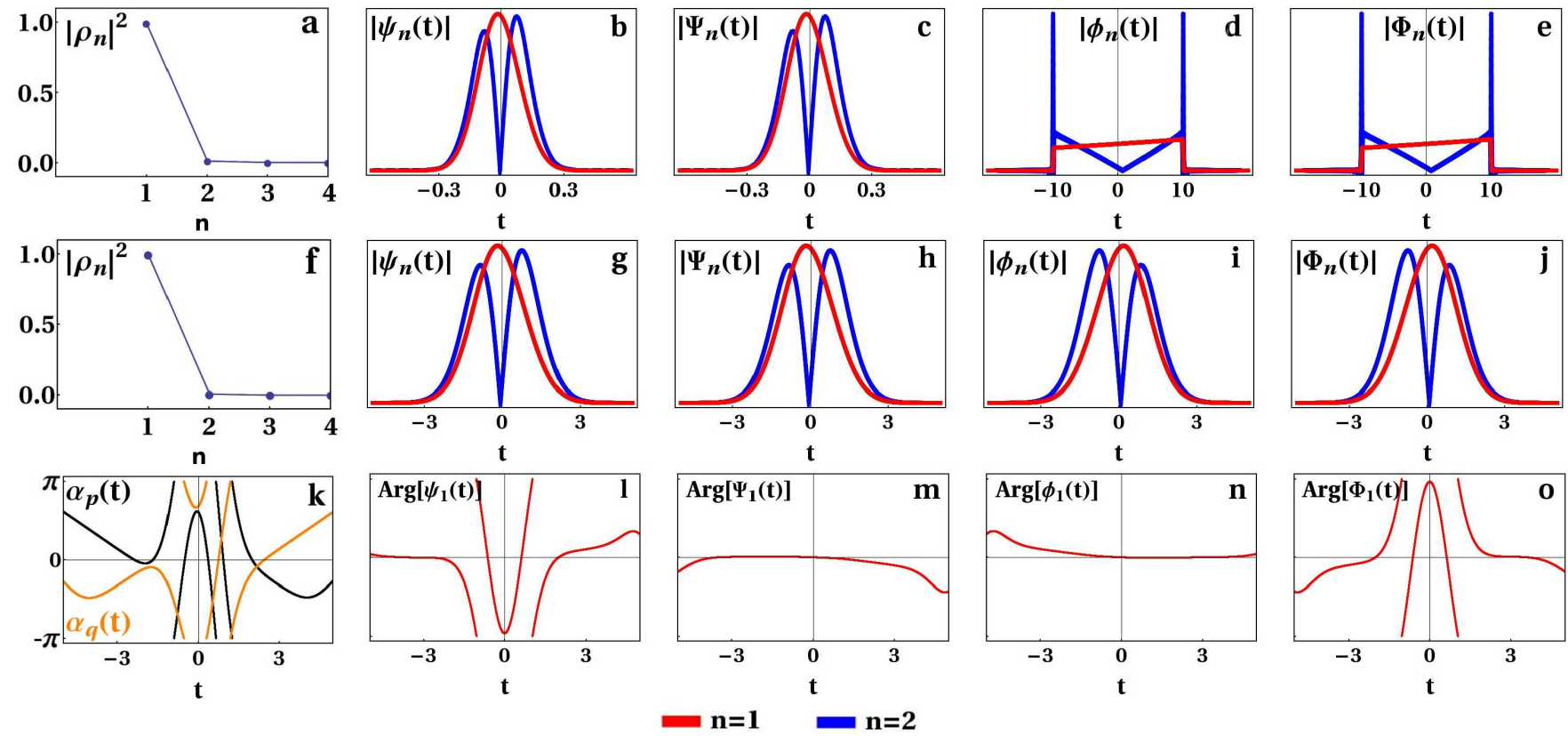

Fig. 3. Conversion efficiencies and Schmidt modes for (a,b,c,d,e) TWM-TMI, (f,g,h,i,j) FWM-TMI, and (l,m,n,o) first Schmidt-mode phase profiles for FWM-TMI, all with Gaussian pumps. Plots (a,f) show the conversion efficiencies of the first four Schmidt modes. The first two Schmidt modes for the corresponding processes are shown for $r$-input $(\mathrm{b}, \mathrm{g}), r$-output $(\mathrm{c}, \mathrm{h}), s$-input $(\mathrm{d}, \mathrm{i})$, and $s$-output $(\mathrm{e}, \mathrm{j})$. (k) shows the first-stage pump pre-chirps used for FWM-TMI.

multiplexing completes the toolkit for encoding information on quantum and classical states of light.

We thank L. Mejling, K. Rottwitt and J. Nunn for helpful discussions. This work was supported by the National Science Foundation through EPMD and GOALI, grant ECCS-1101811.

\section{References}

1. H. Sasada and M. Okamoto, Phys. Rev. A 68, 012323 (2003).

2. T. Yarnall, A. F. Abouraddy, B. E. A. Saleh, and M. C. Teich, Phys. Rev. Lett. 99, 250502 (2007).

3. J. T. Barreiro, T. C. Wei, and P. G. Kwiat, Nature Phys. 4, 282 (2008).

4. G. C. G. Berkhout, M. P. J. Lavery, J. Courtial, M. W. Beijersbergen, and M. J. Padgett, Phys. Rev. Lett. 105, 153601 (2010).

5. N. Bozinovic, Y. Yue, Y. Ren, M. Tur, P. Kristensen, H. Huang, A. E. Willner, and S. Ramachandran, Science 340, 1545 (2013).

6. I. L. Chuang and Y. Yamamoto, Phys. Rev. A 52, 3489 (1995).

7. I. Marcikic, H. de Riedmatten, W. Tittel, V. Scarani, H. Zbinden, and N. Gisin, Phys. Rev. A 66, 062308 (2002).

8. B. J. Smith and M. G. Raymer, New J. Phys. 9, 414 (2007).

9. A. Christ, C. Lupo, and C. Silberhorn, New J. Phys. 14, 083007 (2012).

10. T. Vértesi, S. Pironio, and N. Brunner, Phys. Rev. Lett. 104, 060401 (2010).
11. A. Eckstein, B. Brecht, and C. Silberhorn, Opt. Express 19, 13770 (2011).

12. H. J. McGuinness, M. G. Raymer, C. J. McKinstrie, and S. Radic, Phys. Rev. Lett. 105, 093604 (2010).

13. M. G. Raymer and K. Srinivasan, Physics Today 65(11), 32 (2012).

14. J. Huang and P. Kumar, Phys. Rev. Lett. 68, 2153 (1992).

15. A. P. Vandevender and P. G. Kwiat, J. Mod. Opt. 51, 1433 (2004).

16. M. A. Albota and F. N. C. Wong, Opt. Lett. 29, 1449 (2004).

17. R. V. Roussev, C. Langrock, J. R. Kurz, and M. M. Fejer, Opt. Lett. 29, 1518 (2004).

18. L. Mejling, D. S. Cargill, C. J. McKinstrie, K. Rottwitt, and R. O. Moore, Opt. Express 20, 27454 (2012).

19. C. J. McKinstrie, L. Mejling, M. G. Raymer, and K. Rottwitt, Phys. Rev. A 85, 053829 (2012).

20. A. S. Clark, S. Shahnia, M. J. Collins, C. Xiong, and B. J. Eggleton, Opt. Lett. 38, 947 (2013).

21. A. Christ, B. Brecht, W. Mauerer, and C. Silberhorn, New J. Phys. 15, 053038 (2013).

22. D. V. Reddy, M. G. Raymer, C. J. McKinstrie, L. Mejling, and K. Rottwitt, Opt. Express 21, 13840 (2013).

23. L. E. Myers, R. C. Eckardt, M. M. Fejer, R. L. Byer, W. R. Bosenberg, and J. W. Pierce, J. Opt. Soc. Am. B 12, 2102 (1995).

24. M. G. Raymer, S. J. van Enk, C. J. McKinstrie, and H. J. McGuinness, Opt. Commun. 283, 747 (2010).

25. D. Burnham and R. Chiao, Physical Review 188, 667 (1969). 